\title{
BMJ Open Simulation-based inter-professional education to improve attitudes towards collaborative practice: a prospective comparative pilot study in a Chinese medical centre
}

\author{
Ling-Yu Yang, ${ }^{1,2}$ Ying-Ying Yang, ${ }^{2,3,4}$ Chia-Chang Huang, ${ }^{2,3,4}$ Jen-Feng Liang, ${ }^{1,2}$ \\ Fa-Yauh Lee, ${ }^{2,4}$ Hao-Min Cheng, ${ }^{1,2}$ Chin-Chou Huang, ${ }^{2,3,4}$ Shou-Yen Kao ${ }^{2,4}$
}

To cite: Yang L-Y, Yang Y-Y, Huang C-C, et al. Simulationbased inter-professional education to improve attitudes towards collaborative practice: a prospective comparative pilot study in a Chinese medical centre. BMJ Open 2017;7:e015105. doi:10.1136/ bmjopen-2016-015105

- Prepublication history and additional material for this paper are available online. To view please visit the journal (http:// dx.doi.org/10.1136/bmjopen2016-015105).

Received 8 November 2016 Revised 27 September 2017 Accepted 28 September 2017

\section{(a) CrossMark}

${ }^{1}$ Department of Medical Education, Taipei Veterans General Hospital, Taipei, Taiwan ${ }^{2}$ Faculty of Medicine, National Yang-Ming University, Taipei, Taiwan

${ }^{3}$ Division of Clinical Skills Training, Taipei Veterans General Hospital, Taipei, Taiwan

${ }^{4}$ Department of Medicine, Taipei Veterans General Hospital, Taipei, Taiwan

Correspondence to Dr Ying-Ying Yang; yangyy@vghtpe.gov.tw

\section{ABSTRACT}

Objectives Inter-professional education (IPE) builds inter-professional collaboration (IPC) attitude/skills of health professionals. This interventional IPE programme evaluates whether benchmarking sharing can successfully cultivate seed instructors responsible for improving their team members' IPC attitudes.

Design Prospective, pre-post comparative cross-sectional pilot study.

Setting/participants Thirty four physicians, 30 nurses and 24 pharmacists, who volunteered to be trained as seed instructors participated in 3.5-hour preparation and 3.5-hour simulation courses. Then, participants $(\mathrm{n}=88)$ drew lots to decide 44 presenters, half of each profession, who needed to prepare IPC benchmarking and formed Group 1. The remaining participants formed Group 2 (regular). Facilitators rated the Group 1 participants' degree of appropriate transfer and sustainable practice of the learnt IPC skills in the workplace according to successful IPC examples in their benchmarking sharing. Results For the three professions, improvement in IPC attitude was identified by sequential increase in the post-course (second month, $T_{2}$ ) and end-of-study (third month, $T_{3}$ ) Interdisciplinary Education Perception Scale (IEPS) and Attitudes Towards Healthcare Teams Scale (ATHCTS) scores, compared with pre-course (first month, $\left.T_{1}\right)$ scores. By IEPS and ATHCTS-based assessment, the degree of sequential improvements in IPC attitude was found to be higher among nurses and pharmacists than in physicians. In benchmarking sharing, the facilitators' agreement about the degree of participants'appropriate transfer and sustainable practice learnt 'communication and teamwork' skills in the workplace were significantly higher among pharmacists and nurses than among physicians. The post-intervention random sampling survey (sixth month, $T_{\text {post }}$ ) found that the IPC attitude of the three professions improved after on-site IPC skill promotion by new programme-trained seed instructors within teams.

Conclusions Addition of benchmark sharing to a diamond-based IPE simulation programme enhances participants' IPC attitudes, self-reflection, workplace transfer and practice of the learnt skills. Furthermore, IPC promotion within teams by newly trained seed instructors improved the IPC attitudes across all three professions.

\section{Strengths and limitations of this study}

- This pilot study describes the experiences of a prospective cross-sectional cohort of physicians, nurses and pharmacists who volunteered to receive a serial benchmarking-enhanced diamond-based IPE simulation programme for cultivating them as seed instructors to improve team members' IPC attitude.

- In our IPE programme, IPC benchmarking sharing was implemented to enhance participants' continual motivation to self-reflect and to promote IPC among team members.

- Using IEPS and ATHCTS, our study reveals the significant improvements in participants' motivation and IPC attitude across three professions after receiving training with our new IPE programme.

- Through IPC benchmarking presentation, participants' appropriate transfer and sustainable practice of the learnt IPC skills in the workplace was evaluated.

- Participants' satisfaction with the new programme and the degree of improvement in participants' competencies were not evaluated in our study.

- At this stage, the use of newly acquired knowledge or skills by medical professionals of our institution was not evaluated in our study.

- These results were limited to experience in one institution: the degree to which this can be extrapolated to IPE training in other institutions is not known.

\section{INTRODUCTION}

Inter-professional education (IPE) aims to improve the coordination, communication, teamwork and leadership skills of health pro fessionals by learning with, from and about each other. ${ }^{1}$ Two key families of learning theory, behaviourism and constructivism, were applied to the curriculum design of IPE. ${ }^{23}$ It has been reported that learning theories for 
IPE are not mutually exclusive. In fact, theorists agree that inter-professional learning 'by doing' combined with learner centredness is the key. ${ }^{23}$ Healthcare simulations are recognised as an ideal vehicle for IPE. ${ }^{4}$ Today's patients have complex chronic health issues that need inter-professional collaboration (IPC) in order to deliver well-coordinated, high-quality and patient-centred care. ${ }^{5}{ }^{6}$ Simulation-enhanced IPE helps the development of a health professional's IPC skills and these are very important when managing critical clinical situations. ${ }^{7}$

Baker et al reported that 2-hour cardiac resuscitation/ intravenous access simulation-based IPE prepared medical students, nursing students and junior medical residents for their future as practitioners. ${ }^{8}$ In their study, immediate attitudinal scores and responses by means of an Interdisciplinary Education Perception Scale (IEPS) survey were consistently positive among both medical and nursing students. ${ }^{8}$ Undre et al reported that, using technical and human factors rating scales, trainers and multidisciplinary trainees assessed the crisis scenarios-based simulation training favourably, especially in technical skills. ${ }^{9}$ Paige $e t$ al revealed that in a 3-hour simulation-based interdisciplinary operating room, IPE significantly improved the trainees' self-efficacy teamwork performance in role clarity, anticipatory response, cross-monitoring, team cohesion and interaction. ${ }^{10}$ In Vyas' et al study, using the team-building and inter-professional communications survey, pharmacy students reported that semi-urgent situations simulation-based IPE increased their understanding of professional roles and the importance of inter-professional communication. ${ }^{11}$

In Estis' et al study, using an attitudinal survey, speech language pathology, cardio-respiratory care and nursing students reported that simulation-based IPE enhanced their knowledge of medical professional roles/responsibilities and teamwork skills of caring for tracheostomy patients with speaking valves. ${ }^{12}$ Nevertheless, participants in the Estis et al study suggested that pre-simulation training and more structural interaction during the debriefing phase were likely to enhance the effectiveness of the IPE. ${ }^{12}$ Specifically, Watters et al implemented a debrief diamond, following description-analysis-appplication steps, during a 1-day simulation IPE course. ${ }^{13}$ The standardised debrief diamond was designed to allow high-quality exploration of the non-technical aspects of a simulated scenario. The diamond is a two-sided prompt sheet: the first contains the scaffolding, with a series of constructed questions for each phase of the debriefing, while the second lays out the theory behind the questions and the process. ${ }^{13}{ }^{14}$ In Watters' et al study, using self-efficacy questionnaires, doctors and nurses reported that diamond-based simulation increased their confidence in 'communication and teamwork' skills. ${ }^{13}$ Darlow et al reported that the addition of a preparation workshop to their 11-hour IPE programme resulted in improved attitudes towards inter-professional teams and inter-professional learning, as well as self-reported ability to function within an inter-professional team. ${ }^{15}$

Taken together, previous simulation-based IPE studies ${ }^{8-15}$ were lacking in post-course continuous training. In addition, there is an absence of long-term follow-up that allows the transference and sustainability of IPC practice to be assessed. Furthermore, there is an absence in these studies of opportunities for the participants to reflect on their training after a period of IPC practice. In 2014, a random sampling survey of three professions at our institution revealed that IPC attitudes of physician, nurses and pharmacists need to be improved (figure 1).

It is important to develop feasible continuous IPE/IPC strategies to solve the problems of previous studies ${ }^{8-15}$ and of our survey. Therefore, our education committee targeted these three professions and organised a new IPE programme characterised by pre-simulation training, post-course continuous training and immediate plus delayed IPC attitude assessments. Additionally, a posttraining e-learning platform and IPC benchmarking sharing provide an opportunity for additional/deepening learning of inter-professional problem-solving skills. Benchmarking sharing, a good indicator of organisational seriousness about quality, is a continuous quality improvement approach. Healthcare benchmarking sharing provides opportunities for inter-professional participants to learn from others and develop innovative collaborative clinical care. ${ }^{16} 17$ This pilot intervention intentionally evaluates its impact on cultivating new health professionals as seed instructors to promote IPC within their teams.

\section{METHODS}

\section{Participants and setting}

Between January 2015 and May 2016, we conducted a prospective cross-sectional comparative study at the high-fidelity clinical simulation and interactive learning centre of TVGH: this centre trains around 2500 staff each year. Taipei Veterans General Hospital (TVGH) is a 3000-bed medical centre providing primary and tertiary care to active-duty and retired military personnel and their dependents, and the general public. Meanwhile, TVGH is the teaching hospital for several medical universities in northern Taiwan.

Health professionals having more than 1 year but less than 4 years of clinical work experience were invited to participate in this study. The participants volunteering to be trained $(n=94)$ were invited to join the 2015 pilot benchmarking-enhanced diamond-based IPE simulation courses to improve their IPC attitudes. After excluding six participants due to incomplete questionnaires, a total of 88 individuals were included in this study. They consisted of physicians $(n=34)$, nurses $(n=30)$ and pharmacists $(n=24)$.

Ethical approval (2015-06-017CC) was obtained from the Ethics committee of our institution and care was taken to apply the World Medical Association Declaration of Helsinki principles to the research.

\section{Time points of serial assessments}

After a brief introduction, the participants were asked to complete the online pre-course self-assessment on attitudes to IPC in the pre-course survey $\left(\mathrm{T}_{1}\right)$. Each 


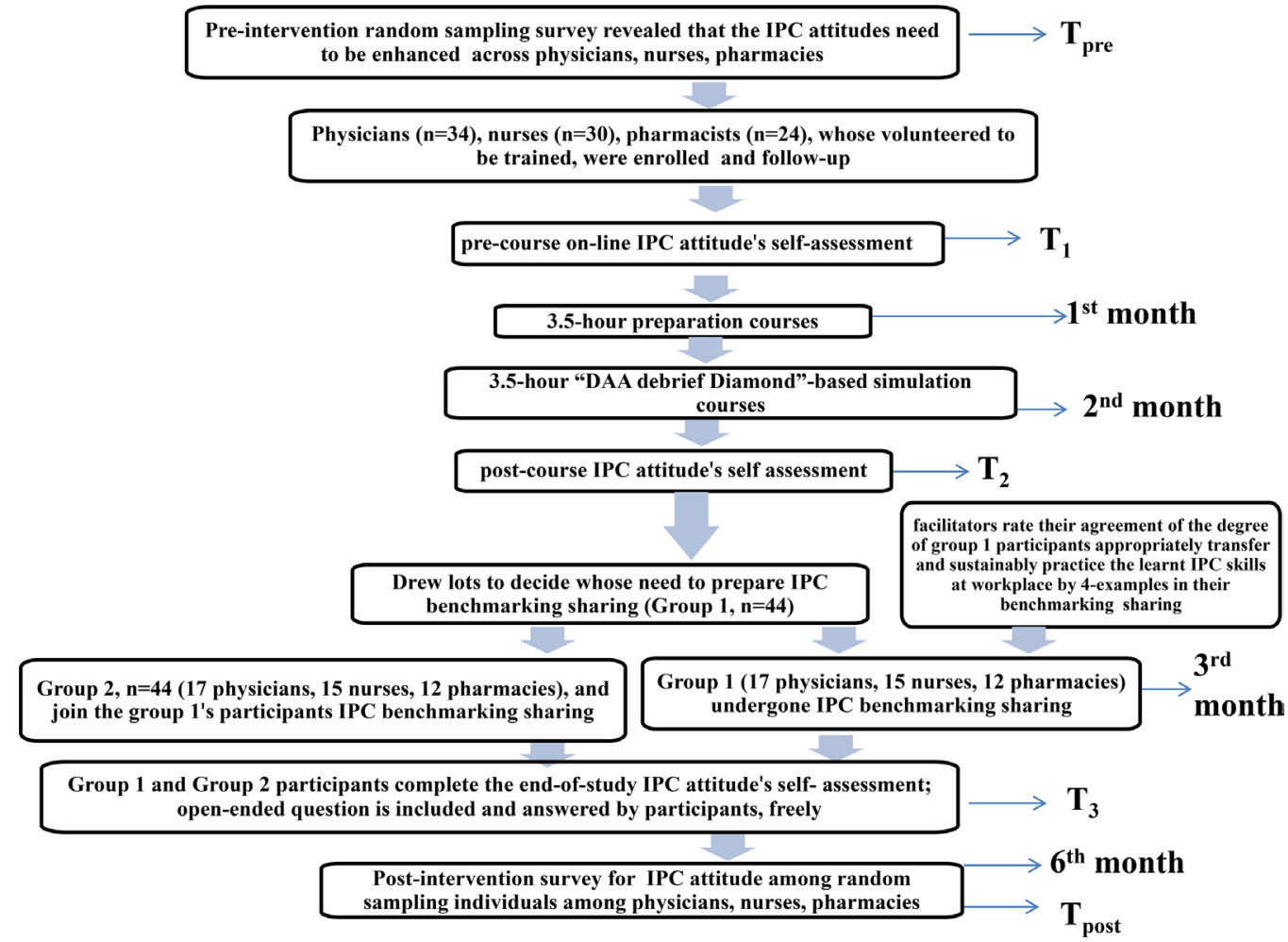

Figure 1 The flow chart of this diamond-based inter-professional education (IPE) simulation study. Detailed time points for training and assessment of this prospective pre-post comparative cross-sectional study.

online self-assessment was numbered so that participants remained anonymous but their numbers could be used to match their pre-course $\left(T_{1}\right)$ self-assessment with post-course $\left(\mathrm{T}_{2}\right)$ and end-of-study $\left(\mathrm{T}_{3}\right)$ self-assessment (figure 1). All participants continued with their usual professional clinical routine throughout the 3-month interventional study.

\section{IPC attitude's self-assessments}

In our study, we measured participants' IPC attitudes with Interdisciplinary Education Perception Scale ${ }^{18-20}$ (IEPS, see online supplement table 1) and the Attitudes Toward Healthcare Teams Scale ${ }^{21}$ (ATHCTS, see online supplement table 2).

Additionally, participants were asked to provide qualitative feedback freely by answering the single-open-ended question, 'What is the one thing you are going to take away with you at the end of this course?' in the online post-courses self-assessment $\left(\mathrm{T}_{3}\right)$. This question was designed to prompt a participant to reflect on their own learning during the course and allowed the programme director to gather evidence on which elements within the courses seemed to be contributing the most to the learning experience.

\section{Benchmarking-enhanced diamond-based IPE simulation courses}

Each participant attended a 3.5-hour preparation course $\left(\mathrm{T}_{1}\right)$ in the first month of this study (figure 1). Subsequently, a 3.5-hour simulation course was arranged for the participants during the second month $\left(\mathrm{T}_{2}\right)$. At the end of the simulation course, participants drew lots to decide who needed to prepare for post-course IPC benchmarking at the third month $\left(\mathrm{T}_{3}\right)$ of study (ie, who was in Group 1 ). In order to maintain a fixed ratio (34:30:24) among the three professions (figure 1), half $(17: 15: 12)$ of the physicians, nurses and pharmacists were selected as Group 1 (benchmarking) and the others as Group 2 (regular).

\section{Facilitators training and DAA debrief diamond}

All facilitators received serial sessions training in how to use the debrief diamond and to gain a consensus on how to rate their agreement about the degree of participants' appropriate transfer and sustainable practice of the trained 'coordination, communication, teamwork and leadership' skills regarding IPC in the workplace using real examples in their benchmarking sharing. In particular, the Description-Analysis-Application (DAA) debrief diamond was used to involve participants in preparation $\left(\mathrm{T}_{1}\right)$ and simulation $\left(\mathrm{T}_{2}\right)$ courses. The 'description' step involved 'description' of each profession's IPC performance in the simulation scenario, along with more challenging 'analysis' and 'application' steps involving 'how did participants feel about each profession's IPC performance in simulation scenario?' and 'how participants may apply the learnt knowledge from IPC simulation scenarios in their own clinical practice'. ${ }^{13} 14$

7-hour preparation and simulation workshop

Preparation courses $\left(T_{t}\right)$

In accordance with previous study design, ${ }^{15}$ two smallgroup preparation workshops were held on two 
Flow chart of activities in each room

\begin{tabular}{|l|l|l|}
\hline $1^{\text {st }}$ month & $\begin{array}{l}\text { In consecutive days, small } \\
\text { group [n=44, with 17:15:12 ratio } \\
\text { of physician: nurse: pharmacists] } \\
\text { 3.5-hour preparation courses } \\
\text { were ran in two rooms, as flow } \\
\text { chart (right-side box show by } \\
\text { arrow head) of activities in each } \\
\text { room }\end{array}$ & Lecture (1-hr) \\
\hline
\end{tabular}

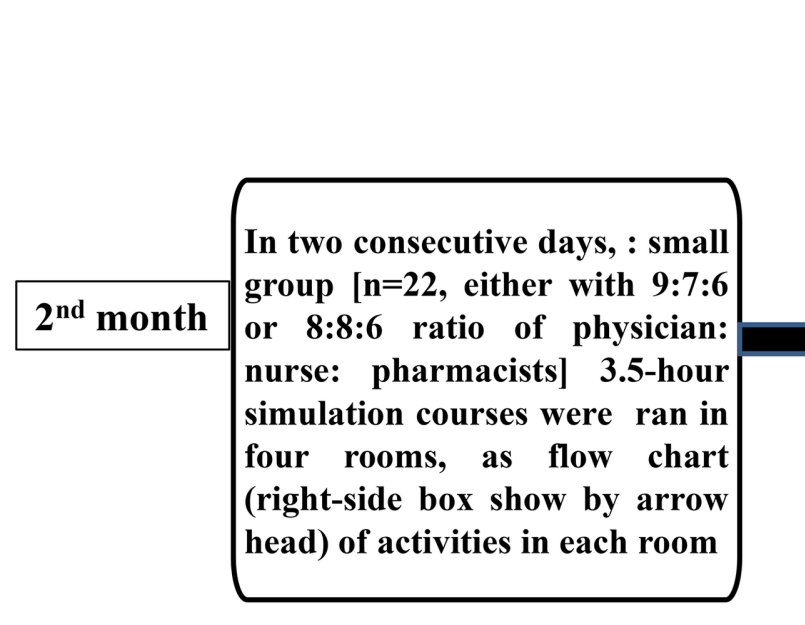

Figure 2 Protocols for small group preparation and simulation workshops. The flow charts and detailed activities of first (preparation) and second (simulation) month' workshops, which were run in separate rooms over two consecutive days.

consecutive days as shown in figure 2. The simulated examples of IPC-based care from a previous study ${ }^{11}$ were revised by an education committee and made into four video clips for IPE. They were, first, a simulation of a distracted wife and a 61-year-old male with dyspnea, who suffered from recurrent asthmatic attacks due to inappropriate home medication; second, a simulation of a 35-year-old pregnant woman and her family, who were anxious as she had nausea/vomiting/abdominal pain and needed anti-emetics suitable for her condition and a paediatrics/gynaecology consultation in an ER setting; third, a simulation of a 57-year-old male with chest pain, with a distraught son and with the wrong allergy and ID labelling on his arm band; and the fourth simulation was an unlocked bed in an ICU setting. These 10-min clips provided a basis for post-video viewing discussions that were led by inter-professional facilitators following a Diamond DAA debriefing of 1 hour. These clips targetted the roles and value of each member of the IPC healthcare team involved in the simulated clinical scenarios presented in the three videos. ${ }^{13} 14$

\section{Simulation courses $\left(T_{2}\right)$}

In our simulation centres, four small-group workshops were held in four rooms within two consecutive days (figure 2). Using the clinical scenario outlined below, workshops were led by well-trained IPE facilitators from
No. of actively involved physicians, nurses, pharmacists $\Rightarrow$ activities

$3: 2: 2=>$ Physicians-led $1^{\text {st }}$ simulation $(\mathbf{0 . 5}-\mathbf{h r})$ for patient' assessment

$3: 2: 2=>1^{\text {st }}$ DAA debrief $(\mathbf{4 0} \mathbf{~ m i n}$. $)$ for $1^{\text {st }}$ simulation by three professions, separately

$3: 3: 2=>$ Pharmacists-led $2^{\text {nd }}$ simulation $(\mathbf{0 . 5}-\mathbf{h r})$ for patient' treatment

$3: 2: 2=>2^{\text {nd }}$ DAA debrief $\left(\mathbf{4 0} \mathbf{~ m i n}\right.$.) for $2^{\text {nd }}$ simulation by three professions, separately

$3: 2: 2=>$ Nurses-led $3^{\text {rd }}$ simulation $(\mathbf{0 . 5}-\mathbf{h r})$ for general care of patient and family

3:3:2 $=>3^{\text {rd }}$ DAA debrief ( 40 min.) for $3^{\text {rd }}$ simulation by three professions, separately, +ending. dietetics, social workers and respiratory therapists. This scenario, which incorporated multi-disciplinary care, was modified in a previous study ${ }^{12}$ and had a practice run before formally being used. A patient scenario involving Mr Jason was developed collaboratively by the faculty members of the aforementioned professions. Participants were given the following information:

Mr Jason has a history of chronic obstructive pulmonary disease (COPD), smokes 60 packs per year of cigarettes and has hypertension, diabetes, coronary artery disease and atrial fibrillation. He has been admitted with an acute exacerbation of his COPD five times over the past year. Home medication includes aspirin, a calcium channel blocker, mycolytic agents, inhalation corticosteroid/bronchodilator (combined) and insulin for subcutaneous administration. MrJason was admitted 3 weeks' ago for emergency coronary artery bypass grafting surgery. Although there has been aggressive management with regular chest theraphy, he has had difficulty being weaned from the ventilator due to poor ability to expectorate sputum and his malnutrition. The primary care teams now are considering a tracheostomy and intensive physical therapy and nutrition therapy. His family members are at the bedside. During the simulation, a pre-set intubated high-fidelity SimMan® 3G simulator acted as the patient and standardised patients (SPs) 
were used as his family. Then, the 3.5 hour courses were run (figure 2).

Before the beginning of the simulation, the participants were presented with the case's name, age, gender, admission diagnosis and current medication/management. In the three simulation phases, the participants involved were expected to carry out assessment, treatments and general care of the patients, collaboratively. Then, the participants began the post-simulation debriefing phase and reflected on the challenges, pitfalls and successes that occurred within the simulation.

The IPC benchmarking $\left(\mathrm{T}_{3}\right)$ of the Group 1 participants

As mentioned above, 17 physicians, 15 nurses and 12 pharmacists formed Group 1 and these participants underwent IPC benchmarking sharing. Presenters were asked to give their four examples of appropriate transfer and sustainable practice learnt IPC skills in the workplace. Randomly, four small groups $(\mathrm{n}=11)$ with ratio $(4: 4: 3$, 4:4:3, 4:4:3 and 5:3:3) of physician to nurse to pharmacists were presented in four rooms over two consecutive days. During benchmarking sharing, two facilitators rated their 5-point Likert-scale-based agreement to the presenters' degree of appropriate transfer and sustainable practice of the learnt IPC skills in the workspace according to their four success examples. In each room, 4 hours $(240 \mathrm{~min})$ were needed for 11 presenters to complete their 20-min presentation $(15 \mathrm{~min}) /$ discussion $(5 \mathrm{~min})$. Each presentation was video-recorded by teaching assistants (TAs) to help with continuous IPC promotion. With the agreement of the presenters, the TAs uploaded edited versions of the video to the e-learning platform. The Group 2 participants were asked to join this end-of-study $\left(\mathrm{T}_{3}\right)$ IPC benchmarking sharing.

\section{e-learning platform}

Both the Group 1 and Group 2 participants were invited to use a common IPE e-learning platform containing the aforementioned scenario, various Power-point presentations, the video used in the preparation/simulation workshop and the video from the IPC benchmarking to encourage self-directed learning.

Pre-intervention ( $\left.\mathrm{T}_{\text {pre }}\right)$ and post-intervention $\left(\mathrm{T}_{\text {post }}\right.$, sixth month) random sampling survey of IPC attitudes

Using IPC core elements-based questionnaires, see online supplement table 3, across the three professions, the effectiveness of the well-trained seed instructors was evaluated by comparing the differences between $\mathrm{T}_{\text {pre }}$ and $\mathrm{T}_{\text {post }}$ IPC attitude scores. ${ }^{22-24}$

\section{Analysis}

Outcomes of our new training programme were analysed according to Kirkpatrick levels. ${ }^{25}$ Since the IEPS and ATHCTS items are ordinal in nature, Wilcoxon's signed rank test was used to analyse each item. The means of the overall IEPS score and the four subscales were evaluated with the Student's two-tailed paired t-test for continuous measures, with the aim of detecting any differences between $\mathrm{T}_{1}$ and $\mathrm{T}_{2}$ as well as $\mathrm{T}_{2}$ and $\mathrm{T}_{3}$ time-points. Data from the IEPS and ATHCTS were matched by profession for analysis with one-way ANOVA or Mann-Whitney U test to detect the significant difference between and/or among groups.

\section{RESULTS}

The baseline characteristics of the participants, including mean age, gender and clinical experiences, were similar across the physicians, nurses and pharmacists as can be seen in table 1 . A higher percentage of pharmacists $(45 \% / 43 \%)$ and nurses $(35 \% / 36 \%)$ had experienced receiving previous IPE training and higher frequency of exposure to IPC meeting during their previous year of clinical works than physicians $(15 \% / 14 \%)$ (table 1$)$. In other words, in comparison with nurses and pharmacists, a lower percentage of physicians belong to the high-exposure ( $>80 \%$ exposure to monthly IPC

Table 1 Baseline characteristics of study population $(n=88)$

\begin{tabular}{|c|c|c|c|}
\hline & $\begin{array}{l}\text { Physicians } \\
(n=34)\end{array}$ & $\begin{array}{l}\text { Nurses } \\
(n=30)\end{array}$ & $\begin{array}{l}\text { Pharmacists } \\
(n=24)\end{array}$ \\
\hline Age (years) & $31.3 \pm 2.7$ & $29.1 \pm 4.8$ & $30.5 \pm 3.6$ \\
\hline \multicolumn{4}{|l|}{ Percentage of distribution of clinical-work-year of participants among groups } \\
\hline $1-2 / 2-3 / 3-4$ years $(\%)$ & $76 / 14 / 10 \%$ & $84 / 10 / 6 \%$ & $69 / 20 / 11 \%$ \\
\hline
\end{tabular}

Percentage of distribution of participants with high/low frequency of exposure

to IPC meeting during their last 1 year of clinical work among groups

\begin{tabular}{lc} 
Percentage of high-exposure participants $†$ & $36 \%$ \\
Percentage of low-exposure participants $\ddagger$ & $43 \%$ \\
\hline
\end{tabular}

${ }^{*} \mathrm{p}<0.01$ versus corresponding nurse's/pharmacist's group.

thigh-exposure participants indicated individual that participatied in more than $80 \%$ of monthly IPC meeting.

flow-exposure participants indicated individual that participatied in less than $20 \%$ of monthly IPC meeting. 
meeting/1 year) group, which indicates the physician's had less experience with IPC meeting participation during their last 1 year of clinical work

\section{Good internal consistency of the IEPS/ATHCTS and its subscales}

In this study, the Cronbach's alpha coefficients of IEPS overall scales (0.721), competency and autonomy subscales (0.69), perceived need for cooperation subscales (0.73), perception of actual cooperation subscales $(0.85)$ and understanding others values subscales $(0.662)$ were good. Meanwhile, Cronbach's alpha of ATHCTS overall scales (0.719), quality of care delivery subscales (0.683), patient-centred care subscales $(0.801)$ and team efficiency subscales $(0.724)$ were acceptable.

\section{Nurses and pharmacists had greater improvement of IEPS and ATHCTS scores than physicians}

The baseline IPC attitude, pre-course $\left(\mathrm{T}_{1}\right)$ IEPS scores and pre-course $\left(\mathrm{T}_{1}\right)$ ATHCTS scores were also similar across the physicians, nurses and pharmacists (table 2). Compared with nurses, there were lower scores for the 'competency and autonomy' and 'understanding others values' basal IEPS subscales $\left(\mathrm{T}_{1}\right)$ among the physicians. Similarly, also compared with nurses, there were lower scores for the 'competency and autonomy' and 'perception of actual cooperation' basal IEPS subscales $\left(T_{1}\right)$ among the pharmacists. Notably, the 'competency and autonomy' subscale of IEPS score and the 'team efficiency' subscale of the ATHCTS score $\left(\mathrm{T}_{2}-\mathrm{T}_{1}\right)$ were increased by the 7-hour stepwise simulation-enhanced IPE course across the three professions. In particular, the magnitude of increase in IEPS and ATHCTS scores were significantly greater among the nurses and pharmacist than among the physicians (table 2). Clearly, pharmacists had greater increase in percentage change of postcourses $\left(\mathrm{T}_{2}\right)$ ATHCTS score from pre-courses $\left(\mathrm{T}_{1}\right)$ score than nurses or physicians (table 2).

Participants demonstrated appropriate transfer of the learnt IPC skills in the workplace and sustainable practice of the skills after training

Based on the real examples in IPC benchmarking presentations of Group 1 participants, the facilitators found that physicians were more appropriately able to transfer and sustainably practice of the learnt IPC 'coordination and leadership' skills in the workspace than pharmacists and nurses (table 3).

In benchmarking sharing, the inter-rater reliability (Kappa statistics) of facilitators for the items used to assess whether participants were able to transfer and sustainably practice the learnt IPC skills was good (table 4).

\section{Participants gave positive descriptive feedbacks to the training}

In open-ended questions at the end of our study, most participants reported that watching the IPE-specific video and discussing it, as well as viewing the uploaded videos on the e-learning platform, markedly encouraged their motivation to improve their IPC attitude. Specifically, the participants reported that having access to an IPE/ IPC-specific e-learning platform was able to improve the users' IPC attitude continuously by providing useful resources and instruction.

Selected completed feedback responses by the participants to the open-ended items of post-course self-assessment $\left(\mathrm{T}_{3}\right)$ are listed below.

1. Benefits of our new benchmarking-enhanced debrief diamond-based IPE simulation courses.

This IPE course improves inter-professional relationships, communication skills, efficiency in holistic patient care and service delivery, team work, respect for one another and builds confidence in my profession.

2. Identified IPE/IPC elements in collaborative training.

We are all geared to patient-centred care, all professions need to use their best assessment and judgement to evaluate patients in order to provide the best patient care that we can.

We understand that there is a lot of team work going on our institution.

We understand that all professions should be encouraged within their training programme to become independent in order to make IPC work better.

3. Improved skills of quality of clinical care.

There are situations that are different, but we do have to rely on the expertise of other professionals in order to obtain the best outcome for the patient.

We were able to collaborate very well with other professional healthcare members, especially with the nurses in their second simulation; they sort of referred to us regarding our drug management skills and sort of learnt how important pharmacists can actually be in a hospital setting.

4. Skills learnt from their skillful facilitators.

Sometimes, staying in your own profession is great and everything, but you really sort of need to reach outwards and see what other professions have to offer, because only if you do that can you truly use the entire knowledge base of other professions and provide the best patient care.

\section{Addition of benchmarking sharing to enhance the continuous beneficial effects of training}

Compared with pre-courses $\left(\mathrm{T}_{1}\right)$ scores, the degree of increase in total IEPS and ATHCTS scores at post-courses $\left(T_{2}\right)$ self-assessments were not different between Group 1 and 2 participants (data not shown). Among the Group 1 and 2 participants, similar or higher end-of-study $\left(\mathrm{T}_{3}\right)$ IEPS and ATHCTS scores than post-courses $\left(T_{2}\right)$ scores indicated the sustained effects of 7-hour simulation-based debrief diamond-enhanced IPE courses (figure 3). From 


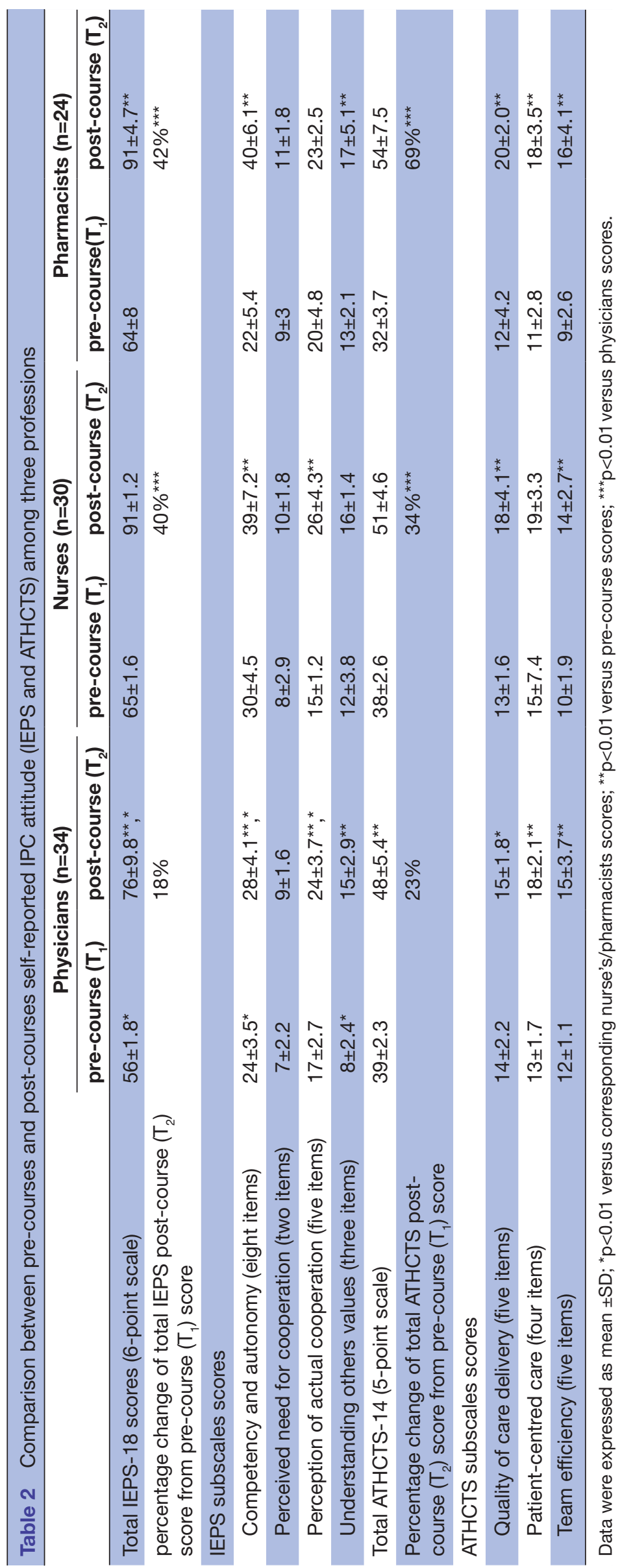


the post-course $\left(\mathrm{T}_{2}\right)$ to end-of-study $\left(\mathrm{T}_{3}\right)$ period, a significantly greater increase in the total IEPS and ATHCTS scores of the Group 1 (benchmarking) participants than for the Group 2 (regular) participants can be seen (figure 3). This indicates the additional benefits of IPC benchmarking on the Group 1 participants' IPC attitude. Among the benchmarking-group participants, the most improved items were the 'competency and autonomy' and 'perception of actual cooperation' subscales of the IEPS and the 'quality of care delivery' and 'team efficiency' subscales of the ATHCTS, when the $T_{2}$ and $T_{3}$ self-assessments were compared.

\section{Improvement of IPC attitudes among team members of three professions by the promotion of new intervention-trained seed instructors}

In total, 132 valid $\mathrm{T}_{\text {post }}$ questionnaires were collected for comparison with another 132 valid $\mathrm{T}_{\text {pre }}$ questionnaires. These anonymous $\mathrm{T}_{\text {pre }}$ and $\mathrm{T}_{\text {post }}$ questionnaires were completed by random members sampled twice from the three professions, namely 51 physicians, 45 nurses and 36 pharmacists. In other words, the individuals who responded to the online IPC attitude survey might be but are not necessarily different between $\mathrm{T}_{\text {pre }}$ and $\mathrm{T}_{\text {post }}$ survey. Nonetheless, it is important to note that the enrolled participants in our interventional study were excluded from the sampling pool for $\mathrm{T}_{\text {post }}$ sampling survey.

Among the randomly sampled team members, the pre-intervention survey $\left(\mathrm{T}_{\text {pre }}\right)$ revealed that IPC attitudes across physicians, nurses and pharmacists must be improved in the aspects of IPC familiarity, understanding of other professions' roles and benefits of IPC on quality of patient-centred care (figure 3C). Across the three professions, after seed instructors began promoting IPC in the workplace, post-intervention ( $\mathrm{T}_{\text {post }}$, sixth month) randomly sampled team members reported that they were familiar with IPC skills, agreed that IPC helped them to understand the role of other team members, agreed that IPC improved patient care quality and agreed that IPC improved team efficiency (figure 3C). Interestingly, across the three professions of randomly sampled team members, the level of agreement to the statement of 'IPC helps provide patient-centred care' were excellent both in the pre-intervention $\left(\mathrm{T}_{\text {pre }}\right)$ and post-intervention $\left(\mathrm{T}_{\text {post }}\right)$ surveys (figure 3C).

\section{DISCUSSION}

In addition to serial subjective and objective assessments, our IPE model is characterised by a debrief diamond strategy (figure 2). Debriefing can help a learner clarify and integrate the simulation experience with their previous knowledge. ${ }^{8} 1011$ 22-24 The debrief diamond encourages a standardised approach to high-quality debriefing across courses, which benefits both the participants and the involved faculty members. ${ }^{13} 14$ The DAA debrief diamond is related to various aspects of the advocacy-inquiry approach and of debriefing with good 
Table 4 Inter-rater reliability of facilitators' ratings in benchmarking sharing of Group 1 participants

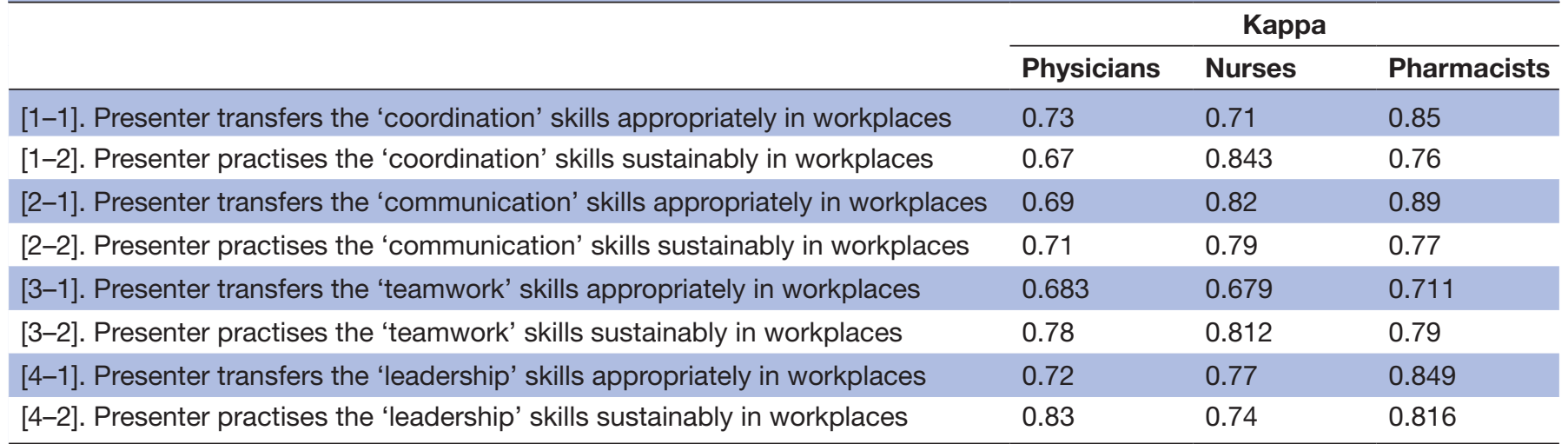

Two facilitators for each small-group ( $n=11$, either with 4:4:3, 4:4:3, 4:4:3, 5:3:3 ratio of physician: nurse: pharmacists) benchmarking sharing held in four rooms over two consecutive days.

judgement. The diamond provides an easy but pedagogically sound structure for facilitators to follow for specific post-simulation feedback and discussion. Nevertheless, the long-term effects of structured debriefing have not been thoroughly evaluated in previous simulated-enhanced IPE studies. ${ }^{8-15}$

When trying to improve each health professional's IPC attitude with limited resources, including the time needed to carry out the training, the number of faculty members needed to run the training and the facilities needed for the training, each newly-trained participant should act as a seed instructor within their team. In other words, successful training of seed instructors can result in profession-wide IPC promotion and attitude remodelling. In our study, this well-organised design allows each participant from three professions to have equal IPE exposure, which helps their development as seed instructors in their healthcare team.
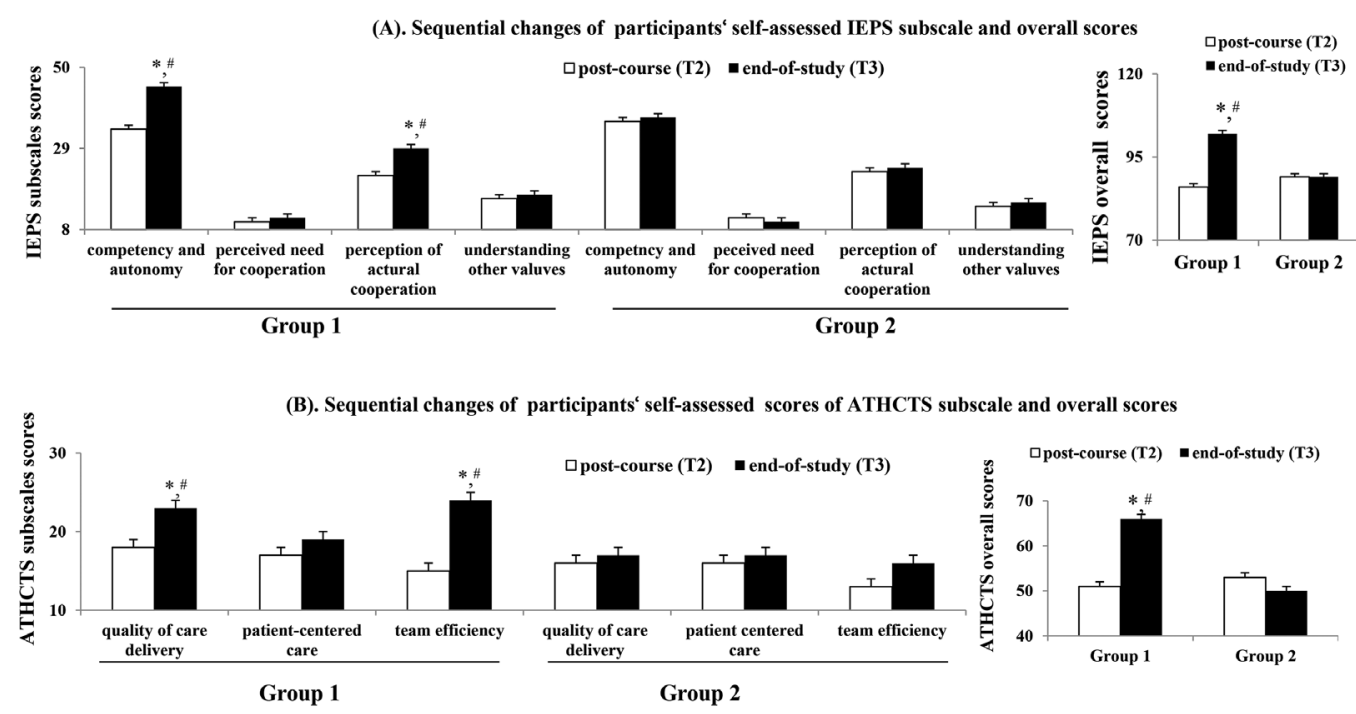

(C) . Randomly sampling survey for the team member's IPC attitudes

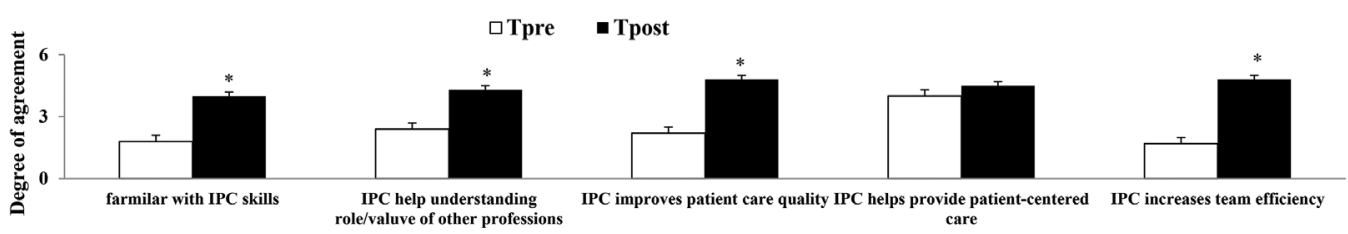

Figure 3 Benchmarking-enhanced IPE pilot programme improved participants and their team members' IPC attitudes. The comparison of sequential changes of post-course $\left(T_{2}\right)$ and end-of-study $\left(T_{3}\right)$ subscales and scales of IEPS (A) and ATHCTS (B) between Group 1 (benchmarking) and Group 2 (regular) participants (C). Comparison of responses from 132 randomly sampled members from the three professions (51 physicians, 45 nurses. 36 pharmacists) about attitudes to IPC in the preintervention $\left(T_{\text {pre }}\right)$ and post-intervention $\left(T_{\text {post }}\right)$ survey. IPC attitude was assessed by five Likert scale responses ranging from 1: strongly disagree to 5 : strongly agree. ${ }^{*} p<0.01$ versus post-course $\left(T_{2}\right)$ or pre-intervention $\left(T_{\text {pre }}\right)$ scores; ${ }^{\#} p<0.01$ versus Group t2 participants' scores. 
By training volunteers from physicians, nurses and pharmacists, our interventional training programme aims to change participants' behaviours and to act as seed instructors for promoting IPC in team member. In our study, the post-intervention survey, performed after the sequential simulation-based IPE courses, revealed that there was significant improvement in randomly sampled team members' IPC attitude across physicians, nurses and pharmacists.

The strengths of our pilot study are the extension of IPE via e-learning platform, benchmarking sharing and continuous self-evaluations. Previous studies have suggested that training videos consistently enhance the observational powers of trainees, as well as improving their ability to integrate different information and increasing their motivation to learn. ${ }^{25}{ }^{26}$ In our study, most participants reported that the availability of an e-learning platform that has sufficient IPE resources helps to continue their self-directed learning. Meanwhile, the benchmarking provides the enrolled participants with the opportunity for IPC self-reflection, as well as enhancing their motivation as seed instructors in their teams.

Primarily, this new simulation-based IPE programme was intended to solve challenges, which included lack of continuous training and follow-up, of previous studies $^{8-1315}$ and those within our institution. Nevertheless, there were some limitations in our study that need to be altered and the method improved for any future study before determining the level of effectiveness of this pilot benchmarking-enhanced debrief diamond-based IPE programme on health professionals IPC practices and outcomes.

For a training programme, Kirkpatrick level 1 and 2 were the evaluation of 'participants satisfaction' and 'participants increase confidence, knowledge and performance'. Using IEPS and ATHCTS, our study revealed significant improvements in participants' motivation and IPC attitudes across the three professions after receiving training with our new IPE programme. The participant's satisfaction with the new programme and the degree of improvement in participant' competency, however, was not evaluated in our study. Kirkpatrick levels 3 and 4 in our study were the 'multiplication' of knowledge by 'seeding' and its influence on the healthcare system. According to the actual case scenario used as the example in benchmarking sharing of our study, facilitators gave high ratings for their level of agreement with the participants' degree of appropriate transfer and sustainable practice of the learnt IPC skills to clinical works. The sequential improvements in participants' self-assessed IPC attitude scores also was noted in our study. Moreover, the comparison of pre-intervention and post-intervention randomly sampled team members, who were non-participants, revealed the general improvement in their IPC attitudes and motivation. Nevertheless, for this part, the use of newly acquired knowledge or skills by medical professionals of our institution was not evaluated in our study. Taken together, our pilot study only achieved some of the goals of a training programme, according to the Kirkpatrick $1-4$ levels. ${ }^{27}$

Our IPE approach targets IPC attitude specifically using a number of defined types of patient scenarios that are suitable for all three of the enrolled professions. Nevertheless, the specific IPC skills required for holistic care of COPD cases clearly are different from those needed to care for acute renal failure cases. Undoubtedly, IPC skills are learnt more readily when the simulation-enhanced IPE used is more relevant to the type of clinical situation. In our study, this limitation was alleviated by the multi-professional post-simulation debrief diamond-based debriefing during a 3.5-hour simulation workshop and the fact that the enrolled participants continued to carry out their regular clinical routines during the 3-month intervention period. In other words, our enrolled participants were likely interacting with other professions in their clinical routine after the first and second stimuli presented during the preparation and simulation workshops. In fact, it has been suggested that learning together with a variety of high-fidelity simulation modules in multi-professional groups would foster shared inter-professional collaborative (IPC) across many clinical situations. ${ }^{28-31}$

As participation in this course was voluntary, participants were likely to be more highly motivated than non-participants, which may limit the generalisability of our results. Actually, the positive effects of the debrief diamond and preparation workshop had been reported in previous simulated-based IPE studies. ${ }^{13-15}$ In our study, the lack of control groups without the debrief diamond method and preparation workshop, to exclude more effects of them on inter-professional skills, may still limit us to conclude the definite effectiveness of benchmarking-enhanced IPE on training. Both IEPS and ATHCTS have been suggested as reliable tools to assess the effectiveness of practice-based IPE interventions. ${ }^{19-21}$ It has been validated that each subscale of IEPS and ATHCTS is a strong measurement for underlying IPC concepts that are crucial to medical professions. ${ }^{19-21}$

Notably, the core elements in the constructive assessment tools, IEPS and ATHCTS, used in our studies were more focused on 'communication and teamwork' than 'coordination and leadership' skills.' Therefore, from table 2, it seems that pharmacists and nurses perform better than physicians. Nonetheless, the facilitators' agreement for the degree of participants' appropriate transfer and sustainable practice of learnt 'coordination and leadership' skills in the workplace were significantly higher among physicians than pharmacists and nurses in benchmarking sharing (table 3). This might be caused by the culture where physicians take over the role of leadership in the healthcare system. These results remind educators to rethink strategies to balance inter-professional training. 


\section{CONCLUSIONS}

Our benchmarking-enhanced debrief diamond-based IPE simulation programme was able to cultivate participants as seed instructors to modify the IPC attitude of their team members. The results of this plot study are promising and suggest that a future large-scale study with extension to professions other than the three professions enrolled here should be considered. As enhancement of inter-professional skills can ensure high-quality patient care, seed instructor training can be suggested as a personal development plan for every health professional.

Acknowledgements The authors express their gratitude to all members of the Clinical Skills Training Centre Department of Medical Education for their input for this article. We also gratefully appreciate Professor Ralph Kirby and Joseph Harwood helping us to correct the English in our manuscript.

Contributors All authors have read and approved the final manuscript. Y-YY conceived and designed the study, analysis and interpretation of data, wrote the draft, recruited the study subjects, planned and performed the statistical analysis. $\mathrm{H}-\mathrm{MC}$ made critical revision of the manuscript. L-YY conceived and designed the study. C-CH recruited the study subjects, analysis and interpretation of data. J-FL acquisition, analysis and interpretation of data. F-YL study supervision, critical revision of manuscript. S-YK study supervision. C-CH acquisition of data. RK critical revision of manuscript.

Funding This work was supported by the government Ministry of Health and Welfare and Taiwan Association of Medical Education (TAME) and V105EA-005 of TVGH from the Taipei Veterans General Hospital, Taipei, Taiwan.

Competing interests None declared.

Ethics approval Ethics committee of Taipei Veterans General Hospital.

Provenance and peer review Not commissioned; externally peer reviewed. Data sharing statement № additional data available.

Open Access This is an Open Access article distributed in accordance with the Creative Commons Attribution Non Commercial (CC BY-NC 4.0) license, which permits others to distribute, remix, adapt, build upon this work non-commercially, and license their derivative works on different terms, provided the original work is properly cited and the use is non-commercial. See: http://creativecommons.org/ licenses/by-nc/4.0/

(c) Article author(s) (or their employer(s) unless otherwise stated in the text of the article) 2017. All rights reserved. No commercial use is permitted unless otherwise expressly granted.

\section{REFERENCES}

1. Frenk J, Chen L, Bhutta ZA, et al. Health professionals for a new century: transforming education to strengthen health systems in an interdependent world. Lancet 2010;376:1923-58.

2. Reeves S, Hean S. Why we need theory to help us better understand the nature of interprofessional education, practice and care. $J$ Interprof Care 2013;27:1-3.

3. Hean S, Craddock D, O'Halloran C. Learning theories and interprofessional education: a user's guide. Learning in Health and Social Care 2009;8:250-62.

4. Pinar G. Simulation-enhanced interprofessional education in health care. Creat Educ 2015;06:1852-9.

5. Deschodt M, Flamaing J, Haentjens $P$, et al. Impact of geriatric consultation teams on clinical outcome in acute hospitals: a systematic review and meta-analysis. BMC Med 2013;11:48.

6. Lapkin S, Levett-Jones T, Gilligan C. A systematic review of the effectiveness of interprofessional education in health professional programs. Nurse Educ Today 2013;33:90-102.

7. Interprofessional Education Collaborative Expert Panel. Core competencies for interprofessional collaborative practice: report of an expert panel. Washington, DC: Interprofessional Education Collaborative, 2001.

8. Baker C, Pulling C, McGraw R, et al. Simulation in interprofessional education for patient-centred collaborative care. J Adv Nurs 2008;64:372-9

9. Undre S, Koutantji M, Sevdalis N, et al. Multidisciplinary crisis simulations: the way forward for training surgical teams. World $\mathrm{J}$ Surg 2007;31:1843-53.

10. Paige JT, Kozmenko V, Yang T, et al. High-fidelity, simulation-based, interdisciplinary operating room team training at the point of care. Surgery 2009;145:138-46.

11. Vyas D, McCulloh R, Dyer C, et al. An interprofessional course using human patient simulation to teach patient safety and teamwork skills. Am J Pharm Educ 2012;76:71.

12. Estis JM, Rudd AB, Pruitt B, et al. Interprofessional simulation-based education enhances student knowledge of health professional roles and care of patients with tracheostomies and Passy-Muir® Valves. $J$ Nurs Educ Practice 2015;5:123-8.

13. Watters C, Reedy G, Ross A, et al. Does interprofessional simulation increase self-efficacy: a comparative study. BMJ Open 2015;5:e005472.

14. Jaye P, Thomas L, Reedy G. 'The Diamond': a structure for simulation debrief. Clin Teach 2015;12:171-5.

15. Darlow B, Coleman K, McKinlay E, et al. The positive impact of interprofessional education: a controlled trial to evaluate a programme for health professional students. BMC Med Educ 2015;15:98

16. Ellis J. All inclusive benchmarking. J Nurs Manag 2006;14:377-83.

17. Ellis J. Sharing the evidence: clinical practice benchmarking to improve continuously the quality of care. J Adv Nurs 2000;32:215-25.

18. Byakika-Kibwika $P$, Kutesa A, Baingana $R$, et al. A situation analysis of inter-professional education and practice for ethics and professionalism training at Makerere University College of Health Sciences. BMC Res Notes 2015;8:598.

19. Luecht RM, Madsen MK, Taugher MP, et al. Assessing professional perceptions: design and validation of an Interdisciplinary Education Perception Scale. J Allied Health 1990;19:181-91.

20. Buelow J, McAdams R, Adams A, et al. Interdisciplinary teamwork: student differences and teaching implications. Am J Health Sci 2010;1:11-21.

21. Heinemann GD, Schmitt MH, Farrell MP, et al. Development of an attitude toward health care teams scale. Eval Health Prof 1999;22:123-42.

22. Reese CE, Jeffries PR, Engum SA. Learning together: using simulations to develop nursing and medical student collaboration. Nurs Educ Perspect 2010;31:33-7.

23. Robben S, Perry M, van Nieuwenhuijzen L, et al. Impact of interprofessional education on collaboration attitudes, skills, and behavior among primary care professionals. J Contin Educ Health Prof 2012;32:196-204.

24. Oandasan I, Reeves S. Key elements of interprofessional education. Part 2: factors, processes and outcomes. J Interprof Care 2005;19(suppl 1):39-48.

25. Chan LK, Patil NG, Chen JY, et al. Advantages of video trigger in problem-based learning. Med Teach 2010;32:760-5.

26. Balslev T, de Grave WS, Muijtjens AM, et al. Comparison of text and video cases in a postgraduate problem-based learning format. Med Educ 2005;39:1086-92.

27. Salvatore VF. Evaluating training programs: The four levels, by Donald L. Kirkpatrick, Berrett-Koehler Publishers. AmJ Eval 1998;19:259-61.

28. Baker C, Medves J, Luctkar-Flude M, et al. Evaluation of a simulation-based interprofessional educational module on adult suctioning using action research. J Res Interprof Pract Educ 2012;2.2:152-67.

29. Luctkar-Flude M, Baker C, Hopkins-Rosseel D, et al. Development and evaluation of an interprofessional simulation-based learning module on infection control skills for prelicensure health professional students. Clin Simul Nurs 2014;10:395-405.

30. Luctkar-Flude M, Baker C, Pulling C, et al. Evaluating an undergraduate interprofessional simulation-based educational module: communication, teamwork, and confidence performing cardiac resuscitation skills. Adv Med Educ Pract 2010;1:59-66.

31. Puskar K, Mitchell AM, Lee H, et al. Simulated case studies illustrate interprofessional education for alcohol and drug use screening for healthcare professionals. ARC J Nurs Healthcare 2016;2:9-20. 